\title{
Gastric Perforation Caused by an Intragastric Balloon: Endoscopic Findings
}

\author{
In Kyung Yoo, Hoon Jai Chun and Yoon Tae Jeen \\ Division of Gastroenterology and Hepatology, Department of Internal Medicine, Institute of Digestive Disease and Nutrition, Korea University \\ College of Medicine, Seoul, Korea
}

Intragastric balloon (IGB) insertion has been most frequently used in the West as an effective endoscopic treatment for morbid obesity, in practice. Recently, there is a growing number of cases requiring IGB deployment for obesity treatment in Korea. One of the reported complications of IGB use is gastric perforation. A 47-year-old woman was admitted to the hospital with mild symptoms, 7 weeks after having an IGB placed. Esophagogastroduodenoscopy was performed and gastric ulcer perforation was observed in the ulcer base, where food particles were impacted. Laparoscopic primary repair was done successfully. This was a case of gastric perforation, secondary to poor compliance with a proton-pump inhibitor (PPI). PPI and Helicobacter pylori eradication are important for ulcer prevention following IGB deployment. Clin Endosc 2017;50:602-604

Key Words: Intragastric balloon; Perforation; Obesity

\section{INTRODUCTION}

Intragastric balloons (IGBs) are gaining popularity as a substitute for bariatric surgery. ${ }^{1}$ Gastric perforation is a rare complication of IGB insertion and requires operative management. However, most serious complications present with obvious signs. We present a case of gastric perforation, 7 weeks after balloon deployment, with almost no complaint of abdominal pain despite food impaction at the perforation site. We also describe the endoscopic findings in gastric perforation caused by an IGB.

Received: January 5, 2017 Revised: April 6, 2017

Accepted: April 7, 2017

Correspondence: Hoon Jai Chun

Department of Internal Medicine, Institute of Digestive Disease and Nutrition, Korea University Anam Hospital, Korea University College of Medicine, 73 Inchon-ro, Seongbuk-gu, Seoul 02841, Korea

Tel: +82-2-920-6555, Fax: +82-2-953-1943, E-mail: drchunhj@chol.com

(c) This is an Open Access article distributed under the terms of the Creative Commons Attribution Non-Commercial License (http://creativecommons.org/ licenses/by-nc/3.0) which permits unrestricted non-commercial use, distribution, and reproduction in any medium, provided the original work is properly cited.

\section{CASE REPORT}

A 47-year-old woman with a body mass index (BMI) of $30 \mathrm{~kg} / \mathrm{m}^{2}$ and a weight of $77 \mathrm{~kg}$ was admitted to the hospital because of mild epigastric pain. She had undergone IGB (Endball) insertion for weight loss 7 weeks prior, but had not been administered a proton-pump inhibitor (PPI), which is necessary after balloon insertion for at least one month. She had no history of other medication intake, such as non-steroidal anti-inflammatory medications, and she had not undergone any previous abdominal surgeries. She had no other known disease. Before presenting to our hospital, she visited another clinic where she had undergone chest radiography, during which a small amount of free air was seen (Fig. 1A). Physical examination showed mild tenderness, and her laboratory test results were unremarkable. Abdominal computed tomography showed the balloon within the stomach, with intraperitoneal free air (Fig. 1B). The provisional diagnosis was gastric perforation. Upper gastrointestinal endoscopy (GIF-Q260J; Olympus, Tokyo, Japan) was performed using carbon dioxide $\left(\mathrm{CO}_{2}\right)$ gas to decompress the balloon and to attempt endoscopic closure of the defect. A $0.8-\mathrm{cm}$ gastric ulcer covered with food particles was noted on the anterior 

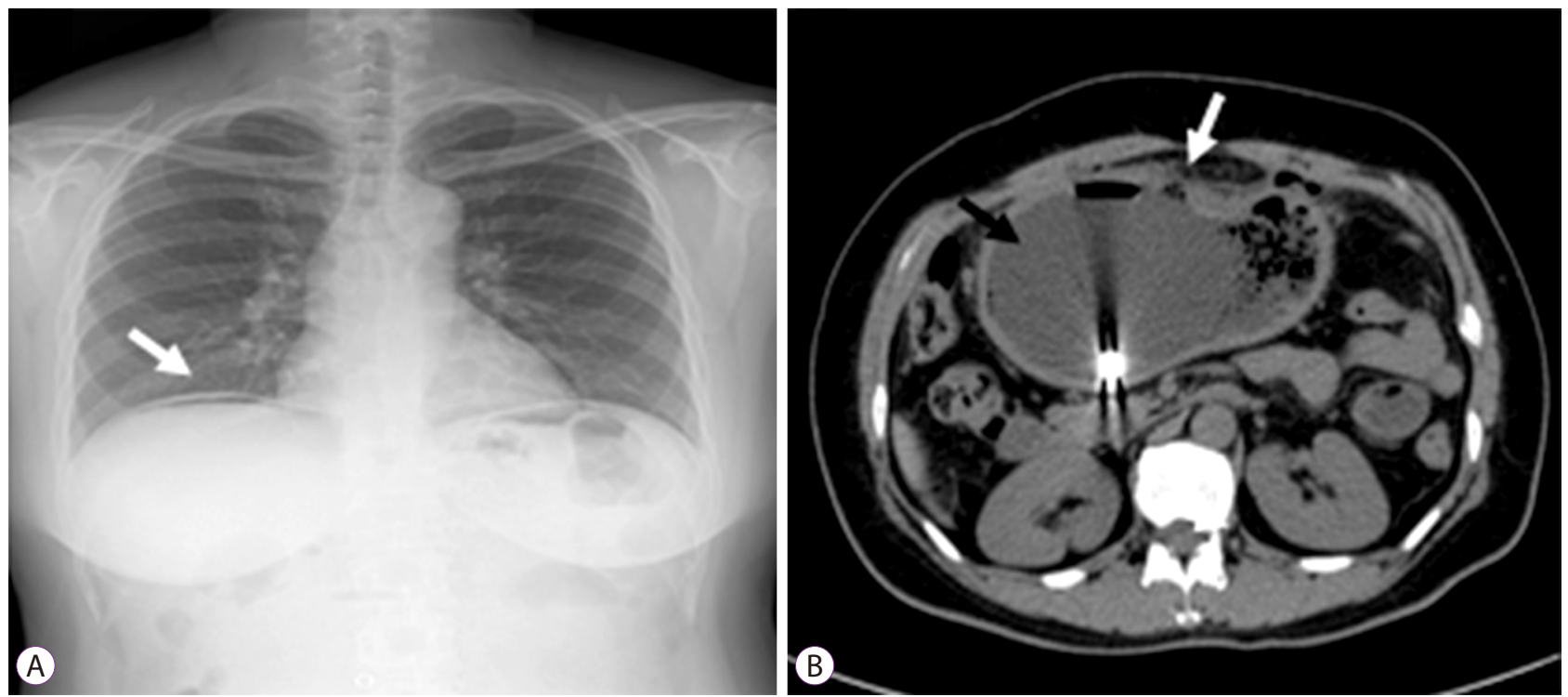

Fig. 1. (A) A small amount of free air was seen on chest radiography (arrow). (B) Computed tomography demonstrating gastric perforation (white arrow) caused by an intragastric balloon (black arrow).

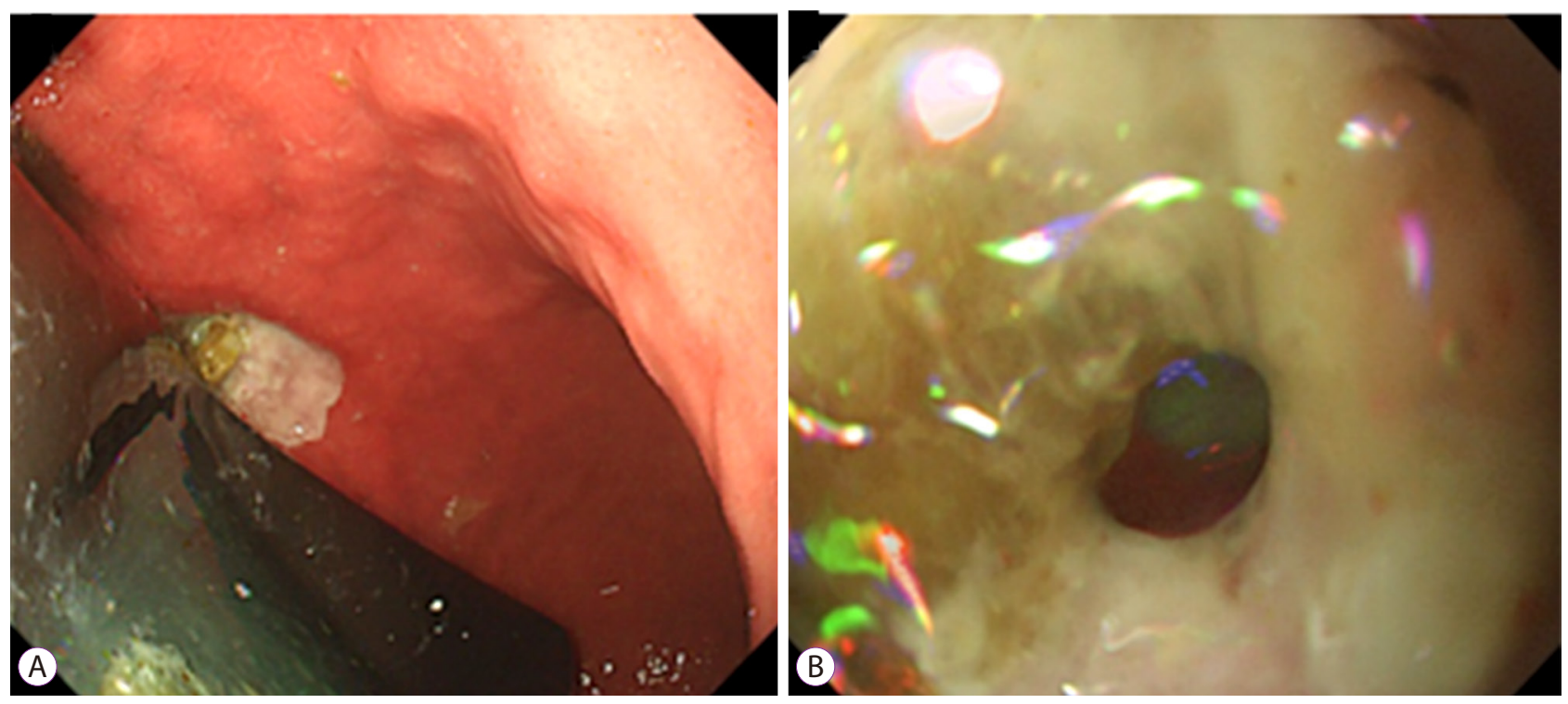

Fig. 2. (A) A gastric ulcer was noted on the anterior wall of the lower gastric body. (B) Definite perforation was observed at the ulcer base after food particles were removed.

wall of the lower gastric body (Fig. 2A). After food particles were removed with a clip device (HX-110LR; Olympus, Tokyo, Japan), a definite perforation was observed at the ulcer base (Fig. 2B, Supplementary Video 1). However, endoscopic closure could not be performed because the ulcer margin showed fibrosis and inflammation. The balloon was punctured and $500 \mathrm{~mL}$ of clear fluid was drained. It was difficult to remove the balloon; therefore, we decided to leave it in the stomach. The patient was transferred to the emergency surgery team. Laparoscopic primary repair was performed, and an omental patch was applied to the perforation. Irrigation and washing of the abdominal and pelvic cavities was done and a hemovac was inserted. Endoscopy was performed 7 days after surgery. The perforation was repaired (Fig. 3). Biopsy for histologic detection of Helicobacter pylori was positive. The eradication of $H$. pylori was then performed. The decompressed IGB was retrieved endoscopically. She was discharged in a stable condition 12 days later and was prescribed PPI. 


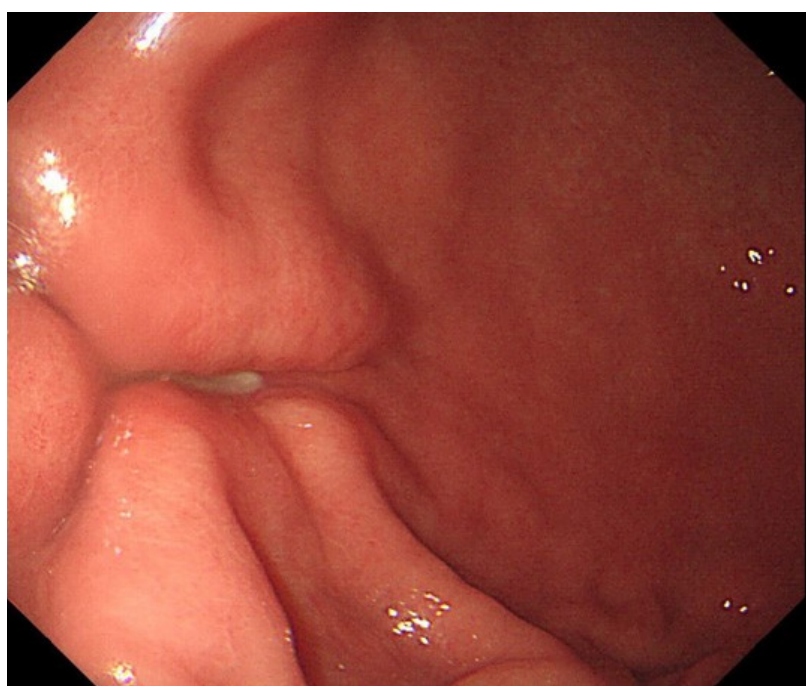

Fig. 3. The follow-up endoscopy showed the repaired perforation.

\section{DISCUSSION}

Recently, IGB insertion has been used as an effective minimally invasive endoscopic treatment for morbid obesity. IGB insertion is popular in Western countries due to the simplicity of the procedure and its applicability to various degrees of obesity. Currently, there is a growing need for IGB deployment to treat obesity in Korea. Common adverse events after IGB placement are pain (33.7\%) and nausea (29\%). Serious adverse events, such as small bowel obstruction, perforation, and death, occur with an incidence of $0.3 \%, 0.1 \%$, and $0.08 \%$, respectively. ${ }^{2}$

Bowel perforation after IGB insertion occurs in $0.1 \%$ of cases. The mechanism of perforation is not well understood. Constant pressure from continuous contact of the balloon with the gastric wall may result in a gastric ulcer and induce perforation. ${ }^{3}$ Complications of balloon insertion constitute a diagnostic challenge because patients sometimes present with non-specific symptoms. ${ }^{4}$ In order to decrease the risk of complications, balloons should not stay in place for more than 6 months ${ }^{5}$ and patients should use PPIs. ${ }^{6}$ Prior gastric surgery resulting in an anatomical abnormality of the gastrointestinal tract is a contraindication for intragastric implantation. ${ }^{7}$ In addition, we recommend $H$. pylori testing and treatment for the prevention of a peptic ulcer. IGB placement is effective and feasible for the management of obesity. However, physicians should be aware of potential complications. As in our case, perforation can present with mild symptoms.

This is a case of gastric perforation secondary to poor compliance with PPIs, and it was evaluated through endoscopy. To our knowledge, this is the first such report. ${ }^{3}$ Although the risk of perforation is low, PPI and $H$. pylori eradication are important for ulcer prevention following IGB deployment.

\section{Conflicts of Interest}

The authors have no financial conflicts of interest.

\section{Acknowledgments}

This research was supported by a grant from the Korea Health Technology R\&D Project through the Korea Health Industry Development Institute, funded by the Ministry of Health \& Welfare, Republic of Korea (grant number: HI14C3477).

\section{Supplementary Material}

Video 1. A gastric ulcer perforation appeared in the ulcer base where food particles were removed with the clip device (https://doi.org/10.5946/ ce.2017.015.v001).

\section{REFERENCES}

1. Evans JD, Scott MH. Intragastric balloon in the treatment of patients with morbid obesity. Br J Surg 2001;88:1245-1248.

2. ASGE Bariatric Endoscopy Task Force; ASGE Technology Committee, Abu Dayyeh BK, et al. Endoscopic bariatric therapies. Gastrointest Endosc 2015;81:1073-1086.

3. Abou Hussein BM, Khammas AA, Al Ani AM, et al. Gastric perforation following intragastric balloon insertion: combined endoscopic and laparoscopic approach for management: case series and review of literature. Obes Surg 2016;26:1127-1132.

4. Nikolic M, Mirosevic G, Ljubicic N, et al. Obesity treatment using a bioenterics intragastric balloon (BIB)--preliminary Croatian results. Obes Surg 2011;21:1305-1310.

5. El Hage Chehade HH, El Khatib ZO, Abtar HK. What could happen if you insert a BioEnterics intragastric balloon after sleeve gastrectomy? Surg Obes Relat Dis 2015;11:e39-e41.

6. Genco A, Bruni T, Doldi SB, et al. BioEnterics intragastric balloon: the Italian experience with 2,515 patients. Obes Surg 2005;15:1161-1164.

7. Espinet-Coll E, Nebreda-Durán J, Gómez-Valero JA, et al. Current endoscopic techniques in the treatment of obesity. Rev Esp Enferm Dig 2012;104:72-87. 